\title{
Massive RBC fragmentation masks severe thrombocytopenia in both impedance and optical platelet count measurements - a case report of a neonate on ECMO support
}

\author{
ANA MLINARIĆ, GORDANA FRESSL JUROŠ, DUNJA ROGIĆ \\ Department of Laboratory Diagnostics, University Hospital Zagreb
}

Corresponding author:

Ana Mlinarić

Department of Laboratory Diagnostics

University Hospital Centre Zagreb

Kišpatićeva 12, 10000 Zagreb, Croatia

Phone: +385989284198

Fax: +385012367395

E-mail:ana.mlinaric@yahoo.com

\begin{abstract}
Extracorporeal membranous oxygenation (ECMO) is a life-saving treatment for paediatric patients with cardiac or respiratory failure of diverse aetiology. ECMO support is implemented only when all other available medications and procedures fail to treat the underlying cause of organ failure. The reason for caution is a high risk of complications, including intravascular haemolysis, bleeding or clot formation, inflammation and sepsis. Platelet count is closely monitored in assessment of bleeding risk associated with ECMO support. Platelets are counted as a parameter of the complete blood count and can be measured using impedance or optical technology. We report a case of severe red blood cell (RBC) fragmentation in a neonate on ECMO in whom erroneously normal platelet counts were obtained by all available automated methods for platelet count in an emergency laboratory. Based on those observations we have implemented an additional procedure for recognising interferences of fragmented and microcytic RBCs that cause spuriously normal platelet counts.
\end{abstract}

Key words: platelet count, ECMO, fragmented $R B C$, neonates, analytical interference

\section{INTRODUCTION}

Extracorporeal membranous oxygenation (ECMO) is a commonly used therapy for a variety of conditions that result in respiratory or cardiac failure. These include persistent pulmonary hypertension, congenital diaphragmatic hernia, meconium aspiration syndrome, respiratory distress syndrome, pneumonia, severe air-leak syndromes, sepsis and septic shock. $(1,2)$ Even though lifesaving, ECMO introduces a high risk of complications including intravascular haemolysis, bleeding or clot formation, inflammation and sepsis. $(3,4)$

Thrombocytopenia in neonates is a major problem in neonatal intensive care units (NICU), as studies have shown the association of thrombocytopenia with increased mortality, length of hospital stay as well as some major comorbidities such as intraventricular haemorrhage, disseminated intravascular coagulopathy and necrotising enterocolitis. A rapid decrease in platelet count is often observed in neonatal patients on ECMO support because of platelet destruction or accumulation in the ECMO circuit. Therefore it is crucial that thrombocytopenia is correctly diagnosed to implement timely therapy. (5)

Platelet count is routinely measured on an automated haematology analyzer as a parameter of the complete blood count (CBC). Platelets are counted using impedance or optical light scatter methods of detection in a routine/emergency setting. In the former, a signal (change in resistance) is generated whenever a cell passes through an orifice connecting two chambers, each containing a differently charged electrode.
Detected signals are presented in the histogram where the size of the change in impedance corresponds to the cell volume. Therefore, the impedance method allows for the differentiation of different cell lineages, such as platelets and red blood cells (RBCs), based on the cell size. $(1,5)$ This is sufficient when platelet and RBC size are normal. However, in cases of abnormal platelet (large platelets) or RBC (microcytes, shizocytes) size, cell fragments, and less commonly, bacteraemia and cryoglobulins, impedance platelet counting is subjected to interferences because of overlap between RBCs and platelets and is therefore inadequate as a method for counting platelets. (6-8) In such cases, optical light scatter platelet measurement serves as a helpful tool for obtaining an accurate platelet count. $(7,9)$ The optical method on the Sysmex haematological analyzer uses a fluorescent dye that binds to ribonucleic acid (RNA) of platelets and reticulocytes. Particles are differentiated based both on fluorescence intensity which is proportionate to the RNA content and on the cell size depending on the detected scattered laser light, as shown in figure $1 \mathrm{f}$.

We report a case of massive RBC fragmentation in a neonate on ECMO support which masked severe thrombocytopenia because of methodological limitations of both impendance and optical measurement of platelet count by an automated method commonly available in clinical laboratories. 


\section{CASE PRESENTATION}

A neonate was admitted to our tertiary university hospital with the diagnosis of congenital diaphragmatic hernia. Surgery was performed to correct the condition. Following surgery, the patient was put on ECMO and was closely monitored for the presence of complications associated with ECMO support in the NICU. Laboratory tests showed intravascular haemolysis (LDH 1970 IU/L, free haemoglobin 1872 $\mathrm{mg} / \mathrm{L})$, anaemia and thrombocytopenia $(26-106 \times 109 / \mathrm{L})$. On the 7 th day of NICU stay the patient's condition worsened with a significant increase in LDH (4300 IU/L) and free plasma haemoglobin $(9614 \mathrm{mg} / \mathrm{L})$ whereas $\mathrm{CBC}$ analysis using the impedance method showed a spuriously normal platelet count (PLT-I $=147 \times 109 / \mathrm{L}$ ) that was inconsistent with the patient's clinical state. Review of the platelet histogram was highly indicative of interference with the platelet count measurement (figure 1a). Compared to the normal platelet histogram (figure 1b), the histogram of our patient showed that platelets were not correctly separated from the RBC population. According to our laboratory's rules, optical analysis was then performed to obtain a more accurate platelet count, resulting in a PLT count of $160 \times 109 / \mathrm{L}$ and an analyzer flag message for the possible presence of RBC fragments. Microscopic examination of a peripheral blood smear was performed to assess the morphological properties of RBCs. RBC fragments and microcytes were confirmed, yet only 0 to 1 platelets were found per $100 x$ optical field (figure 2). Evidently, the blood smear examination confirmed that both platelet counts (impendance and optical) obtained from the haematology analyzer were incorrect. Therefore, manual estimation of the platelet count in the peripheral blood smear was performed using the modified Fonio method. Estimated platelet count (ePC) was calculated using the following equation:

ePC $(109 / \mathrm{L})=$ platelets (to 1000 RBCs) $\mathrm{x}$ RBC (1012/L);

where $\mathrm{ePC}$ is equal to platelets (up to 1000 RBCs in the peripheral blood smear) and multiplied by RBC count (obtained on a haematology analyzer). Estimated platelet count in the presented sample was $27 \times 109 / \mathrm{L}$, which is analytically and particularly clinically significantly differerent compared to the automated count.
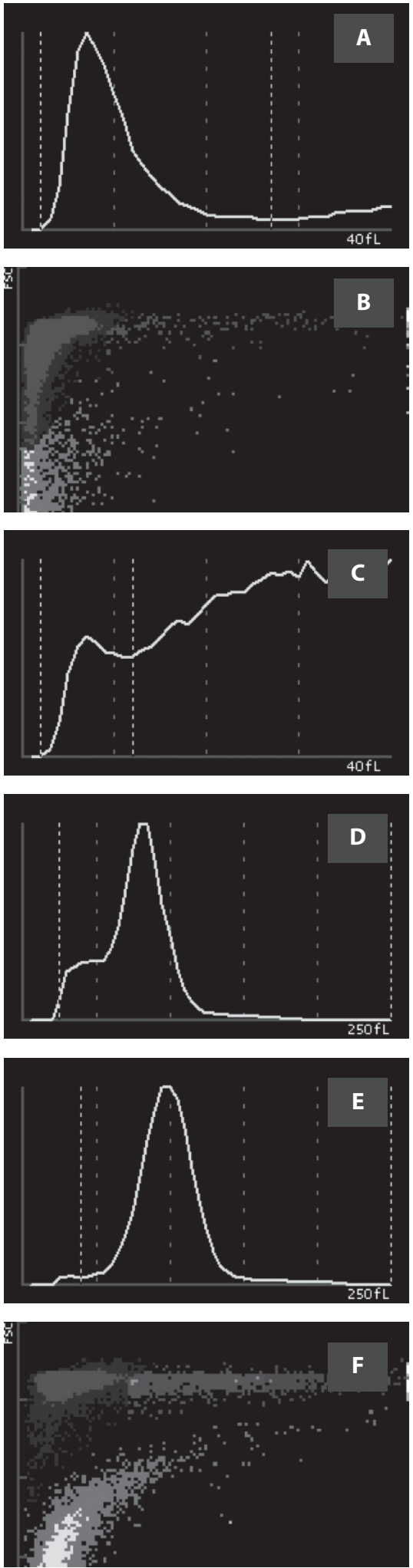

Figure 1. All platelet count measurements were performed on a Sysmex XE 5000 haematology analyzer (Sysmex, Germany): a) platelet histogram shows an overlap at the upper discriminator level which implies that there is an interference in platelet meas- urement; b) normal platelet histogram; c) $R B C$ histogram shows two populations of $R B C$ s indicating the presence of microcytic erithrocytes; d) normal RBC histogram; e) PLT-O scattergram presents a "stretched" $R B C$ population along the forward scatter axis and overlaps with the PLT-O cluster; f) normal PLT-O scattergram.

FSC, forward scatter corresponds to cell size; PLT, platelet count; RBCs, red blood cells (blue), reticulocytes (purple and red), platelets (light blue), immature platelets (green); SFL, side fluorescence corresponds to the RNA content.
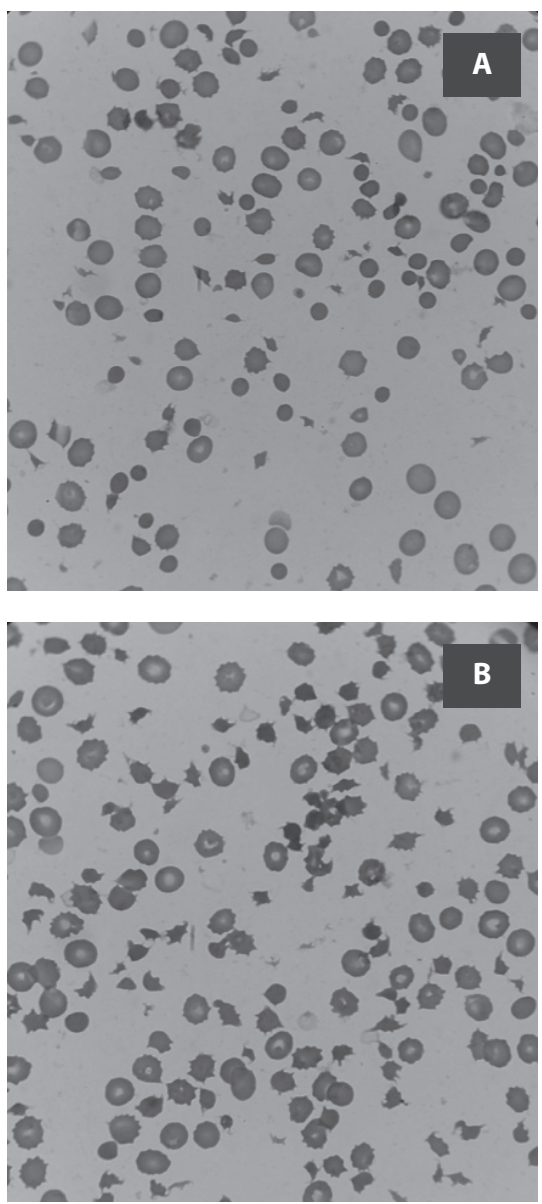

Figure 2. Peripheral blood smear (100x objective, bright field microscopy). A smear was stained with Hemacolor Rapid staining of blood smear (Merck, Germany). Slide review showed anisocytosis, fragmented red blood cells (RBCs) and 0 to 1 platelet per vision field. 


\section{DISCUSSION}

The International Council for Standardisation in Haematology recommends the $\mathrm{RBC} /$ platelet ratio method as the reference method for platelet counting. (10) However, this method is still expensive and largely unavailable for common use in the laboratory setting. The optical method was shown to provide an accurate platelet count in patients with microcytosis. $(5,7)$ Nevertheless, in the presented case, the optical method proved inadequate for platelet counting, which is evident from the PLT-O scattergram, as shown in Figure 1d, and confirmed in the peripheral blood smear (figure 2). $\mathrm{RBC}$ and platelet clusters in the PLT-O scattergram overlap because of the presence of fragmented and microcytic RBCs.
Based on these observations we implemented a review procedure for recognising interferences of fragmented and microcytic RBCs that cause spuriously higher platelet counts. Optical platelet analysis is performed if the distribution of platelet size, measured with the impedance method, in the platelet histogram is abnormal, $i$. e. when there is no visibly clear separation between platelet and RBC population. This abnormality is also indicated by an immeasurable mean platelet volume (MPV) using the Sysmex haematology analyser, and in that case PLT-O scattergram is reviewed. If there is an overlap between PLT$\mathrm{O}$ and RBC clusters, manual review of a peripheral blood smear is performed to check the platelet result obtained from the analyser and to differentiate the source of interference on platelet count. If the microscopic examination confirms an erroneous automated platelet count, manual platelet counting, using the modified Fonio method, is performed and estimated platelet count is reported.

If the interference of fragmented RBCs on optical platelet count had not been recognised in our patient, - and optical platelet count is a counting method that has been otherwise shown to provide accurate platelet counts in cases of microcytosis, - severe thrombocytopenia in a neonate would have been missed, thereby causing a delay in the administration of therapy.

\section{REFERENCES}

1. Chevuru SC, Sola MC, Theriaque DW, Hutson AD, Leung WCW, Perez JA, et.al. Analysis of Platelet Transfusion Usage Among Neonates on Extracorporeal Membrane Oxygenation. Pediatrics 2002;109(6):e89.

2. Bairdain S, Betit P, Craig N, Gauvreau K, Rycus P, Wilson JM, Thiagarajan R. Diverse Morbidity and Mortality Among Infants Treated with Venoarterial Extracorporeal Membrane Oxygenation. Cureus 2015;7(4):e263.

3. Plotz FB, Wildevuur WR, Wildevuur CRH, Delius RE, Bartlett RH. Platelet consumption during neonatal extracorporeal life support (ECLS). Perfusion 1992;7:27-33.

4. Maslach-Hubbard A, Bratton SL. Extracorporeal membrane oxygenation for pediatric respiratory failure: History, development and current status. World J Crit Care Med 2013;2(4):29-39.

5. Ninama NJ, Shah NK. Impedance platelet count in severe microcytosis-study of 161 patients. NHL J Med Scien 2014;3(1):32-6.

6. Zandecki M, Genevieve F, Gerard J, Godon A. Spurious counts and spurious results on haematology analysers: a review. Part I: platelets. Int Jnl Lab Hem 2007;29:4-20.

7. Pan LL, Chen CM, Huang WT, Sun CK. Enhanced Accuracy of Optical Platelet Counts in Microcytic Anemia. Lab Medicine 2014;45(1):32-6.

8. van der Meer W, MacKenzie MA, Dinnissen JWB, de Keijzer HM. Pseudoplatelets: a retrospective study of their incidence and interference with platelet counting. J Clin Pathol 2003;56:772-4.

9. Kunicka JE, Fischer G, Murphy J, Zelmanovic D. Improved Platelet Counting Using Two-Dimensional Laser Light Scatter. Am J Clin Pathol 2000;114:283-9.

10. International Council for Standardization in Haematology Expert Panel on Cytometry and International Society of Laboratory Hematology Task Force on Platelet Counting. Platelet Counting by the RBC/Platelet Ratio Method: A Reference Method. Am J Clin Pathol 2001;115:460-4. 